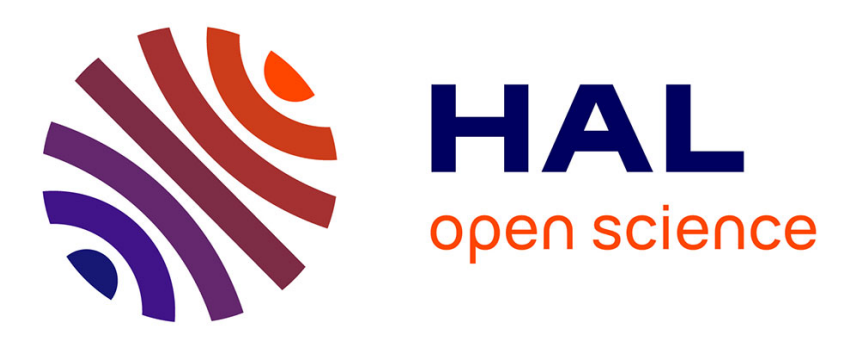

\title{
Collisional dissipation of the laser-induced alignment of ethane gas: Energy corrected sudden quantum model
}

\author{
J.-M. Hartmann, C. Boulet, H. Zhang, F. Billard, O. Faucher, B. Lavorel
}

\section{To cite this version:}

J.-M. Hartmann, C. Boulet, H. Zhang, F. Billard, O. Faucher, et al.. Collisional dissipation of the laser-induced alignment of ethane gas: Energy corrected sudden quantum model. Journal of Chemical Physics, 2018, 149 (21), pp.214305. 10.1063/1.5053963 . hal-01974184

\section{HAL Id: hal-01974184 \\ https://hal.sorbonne-universite.fr/hal-01974184}

Submitted on 8 Jan 2019

HAL is a multi-disciplinary open access archive for the deposit and dissemination of scientific research documents, whether they are published or not. The documents may come from teaching and research institutions in France or abroad, or from public or private research centers.
L'archive ouverte pluridisciplinaire HAL, est destinée au dépôt et à la diffusion de documents scientifiques de niveau recherche, publiés ou non, émanant des établissements d'enseignement et de recherche français ou étrangers, des laboratoires publics ou privés. 


\title{
Collisional dissipation of the laser-induced alignment of ethane gas. Energy Corrected Sudden quantum model.
}

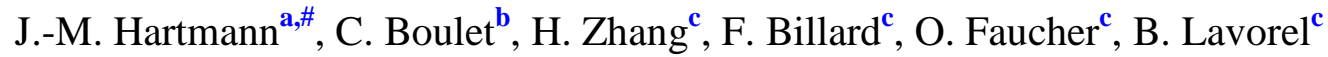 \\ ${ }^{a}$ Laboratoire de Météorologie Dynamique/IPSL, CNRS, École polytechnique, Sorbonne \\ Université, École normale supérieure, PSL Research University, F-91120 Palaiseau, France. \\ \#Corresponding author: jean-michel.hartmann@1md.polytechnique.fr \\ b Institut des Sciences Moléculaires d'Orsay, CNRS, Université Paris-Sud, Université Paris- \\ Saclay, Orsay F-91405, France. \\ 'Laboratoire Interdisciplinaire Carnot de Bourgogne (ICB), UMR 6303 CNRS, Université \\ Bourgogne-Franche Comté, 9 Ave. A. Savary, BP 47 870, F-21078 Dijon Cedex, France.
}

\begin{abstract}
We present the first quantum mechanical model of the collisional dissipation of the alignment of a gas of symmetric-top molecules (ethane) impulsively induced by a linearly polarized nonresonant laser field. The approach is based on use of the Bloch model and of the Markov and secular approximations in which the effects of collisions are taken into account through the state-to-state rates associated with exchanges among the various rotational states. These rates are constructed using the Energy Corrected Sudden (ECS) approximation with (a few) input parameters obtained independently from fits of the pressure-broadening coefficients of ethane absorption lines. Based on knowledge of the laser pulse characteristics and on these rates, the time-dependent equation driving the evolution of the density matrix during and after the laser pulse are solved and the time dependence of the so-called "alignment factor" is computed. Comparisons with measurements, free of any adjusted parameter, show that the proposed approach leads to good agreement with measurements. The analysis of the ECS state-to-state collisional rates demonstrates that, as in the case of linear molecules, collision-induced changes of the rotational angular momentum orientation are slower than those of its magnitude. This explains why the collisional decay of the permanent component of the alignment is significantly slower than that of the amplitudes of the transient revivals in both experimental and computed results. It is also shown that, since intermolecular forces within $\mathrm{C}_{2} \mathrm{H}_{6}$ colliding pairs weakly depend on rotations of the molecules around their C-C bond, the dissipation mechanism of the alignment in pure ethane is close to that involved in linear molecules.
\end{abstract}




\section{INTRODUCTION}

Since detailed and general introductions to the topic of laser-induced molecular alignment can be found in Refs. 1,2, we here only recall studies on the collisional dissipation of the alignment of gases induced by intense and short laser pulses. The pioneering theoretical studies on this subject 3,4 showed that it could be possible to disentangle the effects of rotational population relaxation and pure phase decoherence by using the dissipation of field free alignment. These works, in which a $M$-independent Energy Corrected Sudden (ECS) model was used to construct state-to-state collisional rates for linear molecules, were extended ${ }^{5}$ by introducing an ECS model that explicitly takes into account the dependences on $M$. As confirmed by comparisons with measurements 6,7 taking this dependence into account is essential for correct predictions of the decays of the permanent and transient components of the alignment. Indeed, due to the propensity of collisions to conserve the orientation of the rotational angular momentum ${ }^{5}$, these decays are different, the dissipation of the permanent alignment being significantly slower than that of the transient, a fact not predicted ${ }^{5}$ by the $M$ independent model of Refs. 3,4. Besides the $M$-dependent ECS model, Ref. 5 also proposed, for linear molecules, an original and completely different approach based on requantized Classical Molecular Dynamics Simulations (rCMDS). Its interest was demonstrated by comparisons with experiments 8 and with the predictions of the $M$-dependent ECS model ${ }^{5}$. Besides the above mentioned theoretical studies, experiments probing the collisional dissipation of the laser-induced alignment for linear gaseous molecules can be found in Refs. 6,7,9-11. For non-linear molecules, no results were available under conditions for which collisions play a role until some very recent measurements for ethane ${ }^{12}$ and $\mathrm{CH}_{3} \mathrm{I}^{13}$ and a successful extension of the rCMDS to symmetric-top species ${ }^{14}$. In the absence of a quantum approach for such types of molecules, it is of interest to extend the $M$-dependent ECS model proposed for linear molecules 5 to symmetric tops. This is the subject of the present paper in which comparisons with measurements for ethane gas are also made. Recall that the ECS approach, presented in more details below, enables to explicitly express rotational state-tostate collisional cross sections (or rates) in terms of the relevant quantum numbers and of a limited set of basic collisional quantities (in the case of a linear molecule, the rates $K(J \rightarrow 0)$ for the de-excitation from rotational state $\mathrm{J}$ to $\mathrm{J}=0$ ). This is achieved starting from the Infinite Order Sudden approach that assumes that molecules do not rotate during collisions and correcting for this approximation by introducing "adiabaticity" factors which re-introduce the 
finite energy spacing between rotational states. The resulting model has the advantage of enabling computations for (complex) molecular systems and (elevated) temperatures (such as pure $\mathrm{C}_{2} \mathrm{H}_{6}$ or $\mathrm{CO}_{2}$ near $300 \mathrm{~K}$ ) for which rigorous quantum mechanical treatments (e.g. Close Coupling) require prohibitive computer efforts.

The remainder of this paper is organized as follows: The model is presented in Sec. II together with the associated input data needed for practical computations in the case of ethane gas. Comparisons between measured and computed decays of the laser-induced alignment of pure ethane gas are then presented and discussed in Sec. III before concluding remarks and possible directions for future researches (Sec. IV).

\section{MODEL AND DATA USED}

For reasons explained below, all operators are, or are assumed to be, diagonal versus the rotational quantum number $K$. The model used here for symmetric-top species is thus, except for the state-to-state rates, a quite straightforward extension of that for linear molecules 5 .

\section{A. Time-dependent alignment}

Consistently with what was measured in Ref. 12, we compute the so-called "alignment factor", mean value of the squared cosine of the angle $\theta$ between the molecule symmetry axis (along the $\mathrm{C}-\mathrm{C}$ bond of ethane) and the ( $\vec{Z})$ axis of polarization of the excitation field, i.e.:

$$
\left\langle\cos ^{2}[\theta(t)]\right\rangle=\sum_{J, K, M}\left\langle J K M\left|\boldsymbol{\rho}(t) \cos ^{2}(\theta)\right| J K M\right\rangle
$$

where $\rho(\mathrm{t})$ is the time-dependent density matrix and $J, K$, and $M$ are the rotational quantum numbers. Since the $\cos ^{2}(\theta)$ operator is diagonal in $K$ and $M$, one can write the permanent (P) and transient $(\mathrm{T})$ components of the alignment factor as:

$$
\begin{aligned}
& \left\langle\cos ^{2}[\theta(t)]\right\rangle_{P}=\sum_{J, K, M} \rho_{J M K, J M K}(t)\left\langle J K M\left|\cos ^{2}(\theta)\right| J K M\right\rangle \\
& \left\langle\cos ^{2}[\theta(t)]\right\rangle_{T}=\sum_{J, K, M} \sum_{J^{\prime}} \rho_{J K M, J^{\prime} K M}(t)\left\langle J^{\prime} K M\left|\cos ^{2}(\theta)\right| J K M\right\rangle
\end{aligned},
$$

where $\rho_{J K M, J^{\prime} K M}(t) \equiv\left\langle J K M|\boldsymbol{\rho}(t)| J^{\prime} K M\right\rangle$, and the selection rule associated with $\cos ^{2}(\theta)$ implies that $J^{\prime}=J, J \pm 1, J \pm 2$. 
Starting from a gas at equilibrium, where the density matrix $\rho(t<0)$ before the laser pulse is diagonal with terms given by the Boltzmann relative populations (ie: $\left.\rho_{J K M, J^{\prime} K^{\prime} M^{\prime}}(t<0)=\delta_{J, J^{\prime}} \delta_{K, K^{\prime}} \delta_{M, M} \rho_{J K M}^{0}\right)$, the time dependence is obtained from ${ }^{3,5}$ :

$$
\frac{\mathrm{d} \boldsymbol{\rho}}{\mathrm{dt}}(\mathrm{t})=-\frac{\mathrm{i}}{\hbar}\left[\mathbf{H}_{\mathbf{0}}+\mathbf{H}_{\mathrm{L}}(\mathrm{t}), \boldsymbol{\rho}(\mathrm{t})\right]+\left(\frac{\mathrm{d} \boldsymbol{\rho}(\mathrm{t})}{\mathrm{dt}}\right)_{\text {Coll }},
$$

where $\mathbf{H}_{\mathbf{0}}$ is the free molecule rotation Hamiltonian, which is diagonal with elements given by the rotational energies $E_{J K M}$ of the levels, i.e.:

$$
\left\langle J K M\left|\mathbf{H}_{0}\right| J K M\right\rangle=E_{J K M}
$$

The Hamiltonian $\mathbf{H}_{\mathbf{L}}$, due to the interaction with the linearly polarized laser field $\vec{E}(t)$, is given by:

$$
\mathbf{H}_{\mathbf{L}}(t)=-\frac{1}{2}[\overrightarrow{\overrightarrow{\boldsymbol{\alpha}}} \cdot \vec{E}(t)] \cdot \vec{E}(t)=-\frac{1}{2} \gamma\|\vec{E}(t)\|^{2}\left(\cos ^{2} \theta-1 / 3\right),
$$

in which angle independent terms which do not participate to the dynamics have been removed. In this equation, $\overrightarrow{\overrightarrow{\boldsymbol{\alpha}}}$ is the polarizability tensor of the molecule and $\gamma \equiv \alpha_{\|}-\alpha_{\perp}$ is the anisotropy of the polarizability. As in Refs. 3-5,15, the dissipative dynamics are treated with the Bloch model and the Markov and secular approximations, so that one has, for pure gas at a total pressure $P$ :

$$
\begin{gathered}
\left(\frac{d \rho_{J K M, J K M}(t)}{d t}\right)_{C o l l}=-P\left[\sum_{\left(J^{\prime \prime}, M^{\prime \prime}\right) \neq(J, M)} K_{J, K, M \rightarrow J^{\prime \prime}, K, M^{\prime \prime}}\right] \rho_{J K M, J K M}(t) \\
+P\left[\sum_{\left(J^{\prime \prime}, M^{\prime \prime}\right) \neq(J, M)} K_{J^{\prime \prime}, K, M^{\prime \prime} \rightarrow J, K, M} \times \rho_{J^{\prime \prime} K M^{\prime \prime}, J^{\prime \prime} K M^{\prime \prime}}(t)\right] \\
\left(\frac{d \rho_{J M, J^{\prime} K M^{\prime}}(t)}{d t}\right)_{C o l l}=-\frac{1}{2} \rho_{J K M, J^{\prime} K M^{\prime}}(t) P\left[\sum_{\left(J^{\prime \prime}, M "\right) \neq(J, M)} K_{J, K, M \rightarrow J^{\prime \prime}, K, M^{\prime \prime}}\right. \\
\left.+\sum_{\left(J^{\prime \prime}, M "\right) \neq\left(J^{\prime}, M\right)} K_{J^{\prime}, K, M \rightarrow J^{\prime \prime}, K, M "}\right]-P \gamma_{J K M, J^{\prime} K M}^{P D} \times \rho_{J K M, J^{\prime} K M}(t)
\end{gathered}
$$

Note that the fact (see Sec. II.B) that ethane-ethane collisions only enable $\Delta K=0$ transitions was taken into account in these equations which are thus not fully general. The $K_{J, K, M \rightarrow J^{\prime}, K, M^{\prime}}$ are the pressure-normalized rates of population transfer from state $|J K M\rangle$ to state $\left|J^{\prime} K M^{\prime}\right\rangle$. They include two types of processes: the inelastic ones (in which the value of $J$ changes) as well as those resulting from elastic reorienting collisions (in which the value of $J$ is conserved but that of $M$ changes). $\gamma_{J K M, J^{\prime} K M}^{P D}$ is the pressure-normalized rate of pure 
dephasing of the $|J K M\rangle\left\langle J^{\prime} K M\right|$ coherence which describes the effect of elastic collisions that interrupt the phase of the molecule without quenching it.

\section{B. State-to-state collision-induced rotational transfers}

For linear molecules, the equations for the construction of the $K_{J, M \rightarrow J^{\prime}, M}$, within the Infinite Order Sudden (IOS) approximation were given in Ref. 16. They have been improved by introducing detailed balance and adiabaticity corrections ${ }^{17}$, leading to the so-called Energy Corrected Sudden (ECS) model. The latter has been extensively and successfully applied, in the spectral domain, to the calculation of line-mixing effects on absorption spectra (see Chapt. IV of Ref. 18). It also enabled, in the time domain, accurate descriptions of the dissipation of the laser-induced alignment in $\mathrm{CO}_{2}$ gas 6,7 . A similar procedure was used to build-up an ECS approach for symmetric-top molecules, starting from the IOS model of Ref. 19. Recall that the IOS approach is obtained 18,20 from the Close-Coupling model by first making the Centrifugal Sudden (or Coupled States, CS) approximation, ie: by assuming an effective orbital momentum eigenvalue and freezing the centrifugal potential. Then, starting from the CS equations, the IOS freezes the molecular rotation during each collision, which corresponds to neglecting the energy difference between different rotational states. For molecule-atom collisions, one can then separate the collisional cross -sections into "spectroscopic" and "dynamical" factors in a treatment for symmetric-tops 19 that is analogous to the that given In Refs. 16,21 for linear rotors. The "spectroscopic" terms involve $3 \mathrm{~J}$ and $6 \mathrm{~J}$ symbols which describe the coupling of the involved angular momenta, while the "dynamical" factors are directly related to the expansion of the angle-dependent scattering matrix elements into spherical harmonics. Note that, while the first depend on the final quantity of interest (e.g. state-to-state rotational cross sections, line-coupling among infrared absorption lines, isotropic or anisotropic Raman transitions, etc), the second, that are "basic" collisional quantities (see below), do not. It is then possible to introduce a correction in order to (approximately) take into account the energy difference between rotational states and the finite duration of collisions. This is achieved by introducing 17 a so-called "adiabaticity factor" in the expression of the IOS state-to-state cross sections, which depends on the spacing between adjacent rotational levels and on an effective duration of efficient collisions. In addition, detailed balance corrections are also introduced. Before turning to the state-tostate rotational rates $K_{J, K, M \rightarrow J^{\prime}, K^{\prime}, M^{\prime}}$ involved in the relaxation of the alignment, recall that 
the (spectroscopic) ECS model for symmetric tops enables predictions of the relaxation matrix elements and associated line-mixing process ${ }^{18}$. It was successfully tested by looking at the effects of line-mixing among $M$ components of $\mathrm{CH}_{3} \mathrm{~F}$ lines ${ }^{22}$ (which involve $\Delta J=\Delta K=0$ but $\Delta M \neq 0$ changes) as well as at $\mathrm{NH}_{3}$ spectra at elevated pressures 23,24 (where the absorption shape is sensitive to all possible $\Delta J, \Delta K$ and $\Delta M$ changes). As mentioned above, within the IOS/ECS model, all state-to-state rates (and relaxation matrix elements) can be written 18,19 in terms of basic dynamical factors $Q\left(L, M_{i}, M_{f}\right)$. The latter can be expressed in terms of the products $S_{L, M_{i}}^{l^{*}} S_{L, M_{f}}^{l}$, where * denotes the complex conjugate and $l$ the partial wave, and $S_{L, M}^{l}$ is obtained from the projection of the scattering $\begin{array}{llllll}\text { operator } & \mathbf{S}^{l} \text { into } & \text { spherical } & \text { harmonics } & Y_{L M} & \text { through }\end{array}$ $\left\langle J^{\prime} K^{\prime} M^{\prime}\left|\mathbf{S}^{l}\right| J^{\prime \prime} K^{\prime \prime} M^{\prime \prime}\right\rangle=\sum_{L, M} S_{L, M}^{l}\left\langle J^{\prime} K^{\prime} M^{\prime}\left|Y_{L M}\right| J^{\prime \prime} K^{\prime \prime} M^{\prime \prime}\right\rangle$ [see Eqs. (8) and (14) of Ref. 19]. Note that the $M$-diagonal one $Q(L, M, M)$ is the rate for the collisional de-excitation from the rotational state $J=L, K=M, M$ down to $J=0, K=0, M=0$. It is important to recall that the collisional transitions $J, K, M \rightarrow J^{\prime}, K+\Delta K, M^{\prime}$ with $\Delta K=0$ only result from the contributions of the $Q(L, 0,0)$ while those with $\Delta K \neq 0$ are generated by the $Q\left(L, M_{i} \neq 0, M_{f} \neq 0\right)$ factors ${ }^{19}$. Indeed, this enables to greatly simplify the model in the particular case of pure ethane since, for this system, analysis of a reliable ab initio potential energy surface 25 shows that intermolecular forces are little sensitive to rotations of the molecules around their symmetry (C-C) axes (as confirmed by the results of Ref. 14). As a result, $\Delta K \neq 0$ transitions are very unlikely and assuming that only the $Q(L, 0,0)$ rates make a significant contribution is thus a very reasonable approximation in the case of $\mathrm{C}_{2} \mathrm{H}_{6}-\mathrm{C}_{2} \mathrm{H}_{6}$ collisions. The whole problem then becomes diagonal in $K$ and the IOS/ECS expressions of the rates are simple extensions of those for linear molecule, as detailed below.

Within the ECS model retained here (i.e. assuming $\Delta K=0$ ), the pressure-normalized rates $K_{J, K, M \rightarrow J^{\prime}, K, M}$, for downward collisional transitions (i.e. $\mathrm{J} \geq \mathrm{J}^{\prime}$, where $J, K, M$ denote the rotational quantum numbers) are computed from the following expression: 


$$
\begin{aligned}
K_{J, K, M \rightarrow J^{\prime} \leq J, K, M^{\prime}=\left(2 J^{\prime}+1\right)(2 J} & +1) \sum_{L \text { even } \neq 0}\left\{\left(\begin{array}{ccc}
J & J^{\prime} & L \\
M & -M^{\prime} & M^{\prime}-M
\end{array}\right)^{2}\right. \\
& \left.\times\left(\begin{array}{ccc}
J & J^{\prime} & L \\
K & -K & 0
\end{array}\right)^{2} \frac{\Omega(J)}{\Omega(L)}(2 L+1) Q(L, 0,0)\right\}
\end{aligned}
$$

where (:::) is a $3 \mathrm{~J}$ symbol, $\Omega(J)$ is the adiabaticity correction 17 discussed below and $\left|\mathrm{J}-\mathrm{J}^{\prime}\right| \leq \mathrm{L} \leq \mathrm{J}+\mathrm{J}^{\prime}$. Note that the fact that only even $L$ values are kept in Eq. (7) is an approximation justified by the symmetry of the ethane molecule (which has no dipole moment) and the fact that the quadrupole-quadrupole interaction makes a significant contribution to intermolecular forces in $\mathrm{C}_{2} \mathrm{H}_{6}$ pairs. As done for linear molecules ${ }^{5}$, the ECS rates for upward collisional changes are deduced from those computed using Eq. (7) by using the detailed balance relation, i.e.:

$$
K_{J^{\prime}<J, K, M^{\prime} \rightarrow J, K, M}=K_{J, K, M \rightarrow J^{\prime}, K^{\prime}, M} \times\left(\rho_{J K M}^{0} / \rho_{J^{\prime} K M^{\prime}}^{0}\right),
$$

where $\rho_{J K M}^{0}$ is the equilibrium relative population of state $|J K M\rangle$. The terms $\Omega(J)$ and $\Omega(L)$, which are introduced 17 in order to take into account the rotation of the molecule during collisions, are given in terms of a "scaling length" $\ell_{c}$ (or, alternatively, a characteristic collision duration $\tau_{\mathrm{c}}=\ell_{\mathrm{c}} / \overline{\mathrm{v}}_{\mathrm{r}}$ with $\overline{\mathrm{v}}_{\mathrm{r}}$ the mean relative speed) by:

$$
\Omega(J)=\left[1+\left(\omega_{\mathrm{J}, \mathrm{J}-2} \ell_{\mathrm{c}} / \overline{\mathrm{v}}_{\mathrm{r}}\right)^{2} / 24\right]^{-2},
$$

where $\omega_{J, J-2}=\left(E_{J K M}-E_{J-2 K M}\right) / \hbar$ is obtained from the energy difference between rotational level $J$ and the nearest inferior level significantly coupled to $J$ by the intermolecular potential [i.e. $J-2$ by consistency with the above mentioned fact that only even values of $L$ are retained in Eq. (7)]. The basic quantities $Q(L, 0,0)$ of this model are the rates for the deexcitation from level $J, K, M=L, 0,0$ to $J, K, M=0,0,0$. They can be calculated from the intermolecular potential but they are more generally 18 modeled using an analytical function whose parameters are deduced from fits of measured data. Let us emphasize that Eqs. (7)-(9) enable to calculate the effects of both inelastic and elastic reorienting collisions since they can be used to predict the $K_{J, K, M \rightarrow J^{\prime}=J, K, M^{\prime} \neq M}$.

Finally, the expression of the pure dephasing rates $\gamma_{J K M, J^{\prime} K M}^{P D}$ within the IOS approximation, derived as done for a linear molecule in Ref. 5, is: 


$$
\begin{gathered}
\gamma_{J K M, J^{\prime} K M}^{P D}=\frac{1}{2} \sum_{L \text { even } \neq 0}(2 L+1)\left[(2 J+1)\left(\begin{array}{ccc}
J & J & L \\
-K & 0 & K
\end{array}\right)\left(\begin{array}{ccc}
J & J & L \\
M & -M & 0
\end{array}\right)\right. \\
\left.-\left(2 J^{\prime}+1\right)\left(\begin{array}{ccc}
J^{\prime} & J^{\prime} & L \\
-K & 0 & K
\end{array}\right)\left(\begin{array}{ccc}
J^{\prime} & J^{\prime} & L \\
M & -M & 0
\end{array}\right)\right]^{2} Q(L, 0,0)
\end{gathered}
$$

It is important to recall that the ECS and IOS models, as written here and widely used for other studies 18 , neglect the influence of the rotational structure of the collision partner (perturber). Strictly speaking they are thus only applicable to molecule-atom interactions. As for pure $\mathrm{CO}_{2} 5$ this limitation is, for ethane, of small consequences thanks to the small value of the rotational constant $B$ which makes resonance or non resonance effects between rotational changes of the pair of colliding partners relatively small. This implies that adjacent $J$ levels are close to each other so that these effects, which are associated with the molecular nature of the collisional partner (i.e. its rotational energy structure), are weak. Furthermore, the influence of transfers between rotation and translation, which result in changes of the translational distribution with time after excitation of the rotation by the laser pulse in the alignment experiment, are disregarded in the ECS model. However, the latter remains applicable here since, thanks to the (moderate) laser-pulse energies in the experiments 12,26 used below for the test of the model and to the small value of the anisotropic polarizabiliy of ethane. These make the increase of the rotational energy induced by the interaction of ethane molecules with the excitation field relatively small. The collision-induced rotation-translation transfers thus only slightly change the translational motion and have very small effects on the decay with time of the alignment (see Ref. 5 for a discussion of this issue in the case of $\mathrm{CO}_{2}$ which has a much larger anisotropic polarizabiliy).

\section{Data used and computational procedure}

The data needed for calculations include the energies $E_{J K M}$ and the equilibrium relative populations $\rho_{J K M}^{0}$ of the rotational levels, the $\mathrm{C}_{2} \mathrm{H}_{6}$ anisotropy of the polarizability, the characteristics of the laser pulse, and the parameters of the ECS model [the $Q(L, 0,0)$ for Eqs. (8) and (10) and $\ell_{\mathrm{c}}$ for Eq. (9)]. The values of $E_{J K M}$ and $\rho_{J K M}^{0}$ were computed from:

$$
E_{J K M}=B J(J+1)+(A-B) K^{2}-D_{J}[J(J+1)]^{2}-D_{J K} J(J+1) K^{2}-D_{K} K^{4},
$$

and 
$\rho_{J K M}^{0}=g_{J K M} \exp \left(-E_{J K M} / k_{B} T\right) /\left[\sum_{J, K, M} g_{J K M} \exp \left(-E_{J K M} / k_{B} T\right)\right]$,

where $T$ is the temperature, $g_{J K M}$ is the degeneracy of the $J K M$ level, $A$ and $B$ are the rigid rotor rotational constants and $D_{J}, D_{J K}$ and $D_{K}$ describe the effect the centrifugal distortion. Their values were taken from Ref. 12 together with that of the anisotropy of the $\mathrm{C}_{2} \mathrm{H}_{6}$ polarizability $\left(\gamma=0.7 \mathrm{~A}^{3}\right)$. For the laser pulse, we used the parameters reported in the experimental studies 12,26 .

As widely done in various studies of molecular collisions 18,27 , the basics rates $Q(L, 0,0)$ of the ECS model have been represented by the exponential-power law:

$$
Q(L, 0,0)=Q_{0}[L(L+1)]^{-\alpha} e^{-\beta E_{L 00} / k_{B} T} .
$$

The values of the final set of ECS parameters $\left(Q_{0}, \alpha, \beta\right.$, and $\left.\ell_{\mathrm{c}}\right)$ at room temperature have been obtained from fits of the pressure-broadening coefficients of lines of ethane. In the absence of data for isotropic Raman lines, we used values for infrared ${ }^{\mathrm{R}} \mathrm{Q}-\mathrm{lines}{ }^{28}$ and wrote:

$$
\begin{aligned}
\gamma_{v^{\prime}, J, K \rightarrow v^{\prime}, J, K+1} \simeq(2 J+1)^{-1} \sum_{M} & \frac{1}{2}\left[\sum_{\left(J^{\prime}, K, M^{\prime}\right) \neq(J, K, M)} K_{J, K, M \rightarrow J^{\prime}, K, M^{\prime}}\right. \\
& \left.+\sum_{\left(J^{\prime}, K, M^{\prime}\right) \neq(J, K, M)} K_{J, K+1, M \rightarrow J^{\prime}, K+1, M^{\prime}}\right],
\end{aligned}
$$

in which $\gamma_{v^{\prime}, J, K \rightarrow v^{\prime}, J, K+1}$ is the pressure-broadening coefficient of the $v^{\prime \prime}, J, K \rightarrow v^{\prime}, J, K+1{ }^{\mathrm{R}} \mathrm{Q}$ optical transitions (v" and v' denoting vibrational quantum numbers). Figure 1 shows that a quite satisfactory fit is obtained with the following values of the ECS model parameters:

$$
Q_{0}=44.210^{-3} \mathrm{~cm}^{-1} / \mathrm{atm}\left(8.32 \mathrm{~ns}^{-1} / \mathrm{atm}\right), \alpha=0.87, \beta=0.009 \text { and } \ell_{c}=1.4 \mathrm{~A},
$$

that we have retained for all computations. 


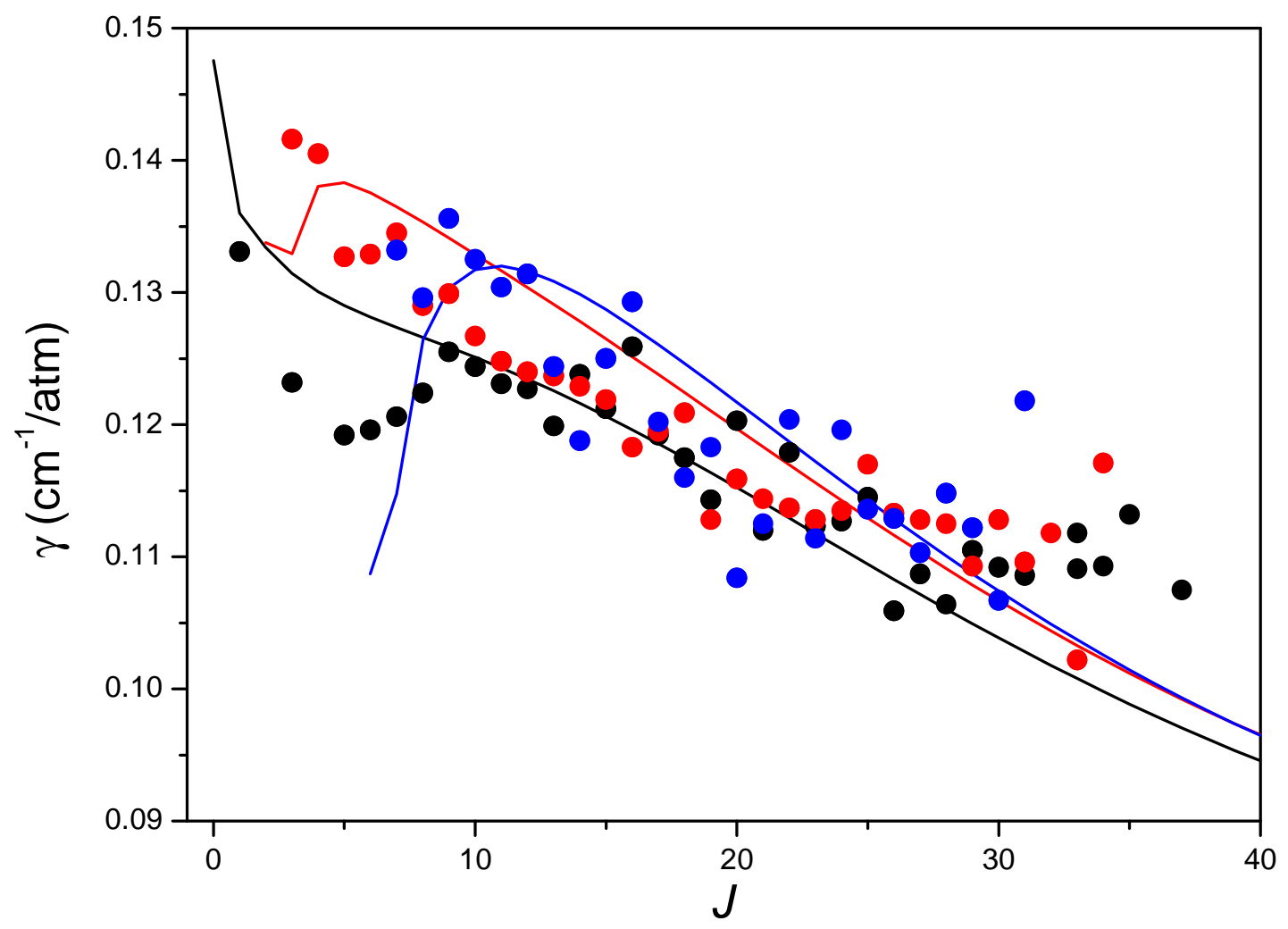

Fig. 1: Measured self-broadening coefficients for the $\gamma_{v^{\prime \prime}, J, K \rightarrow v^{\prime}, J, K+1}{ }^{\mathrm{R}} \mathrm{Q}(J)$ lines of the $v_{9}$ band of pure ethane 28 (symbols) and those computed using Eqs. (7)-(9), (13)-(15) (lines). The results in black, red and blue are for $K=0, K=2$ and $K=6$, respectively.

In the calculations, the conservative values of $J_{\max }=\left|K_{\max }\right|=\left|M_{\max }\right|=50$ for rotational states and a time step of 5 fs [to solve Eq. (3)] were used. For comparisons with experiments and as done in Ref. 12, the computed alignment factor versus time was convoluted by a Gaussian function (FWHM of $150 \mathrm{fs}$ ) which takes into account the finite duration (100 fs) of the probe pulse and the effect of the crossing angle between the two beams. Since the first excited vibrational state $\left(v_{4}\right)$ is significantly populated at room temperature $(25 \%)$, two calculations of the alignment factor were made using the rotational constants of the ground and $\mathrm{v}_{4}=1$ states. For comparisons with measurements, they were then combined, as done in Ref. 12, using: $\left\langle\cos ^{2}[\theta(t)]\right\rangle=0.75\left\langle\cos ^{2}[\theta(t)]\right\rangle^{\text {Ground }}+0.25\left\langle\cos ^{2}[\theta(t)]\right\rangle^{\mathrm{v}_{4}=1}$. Finally note that comparisons of the ECS state-to-state rates $K_{J, K, M \rightarrow J^{\prime}, K, M^{\prime}}$ [Eq. (7)] with the pure dephasing rates $\gamma_{J K M, J ' K M}^{P D}[$ Eq. (10)] show that the latter are more than one order of magnitude smaller, as 
in the case of pure $\mathrm{CO}_{2}{ }^{5}$. Their contribution was thus disregarded and only the $K_{J, K, M \rightarrow J^{\prime}, K, M}$ ' were kept in Eq. (6).

\section{RESULTS AND COMPARISONS WITH MEASUREMENTS}

\section{A. Dissipation of the transient alignment}

A comparison between the measured alignment factor for pure ethane gas at $P=0.69$ bar and initially at $295 \mathrm{~K}^{26}$ and those computed with the ECS approach at the same pressure $P$ and for collision-free conditions ( $\mathrm{P}=0$ bar), are plotted in Fig. 2 for a broad range of delays $\Delta t$ after the pulse. Note that, in the birefringence experiments of Refs. 12 and 26, the probe laser measures the change $\Delta n(\Delta t)$ of the refractive index that is induced by the pump pulse. This change is proportional 12 to the so-called alignment factor $\left\langle\cos ^{2} \theta(\Delta t)-1 / 3\right\rangle$ but the proportionality factor leading to the signal delivered by the photodiodes in the experiment is not known. The results are thus presented in arbitrary units and the calculated results in Figs. $2 \mathrm{a}$ and $2 \mathrm{~b}$ have been multiplied by a constant for the best match with the measured amplitude of the first revival in Fig. 2c. As can be seen, the influence of collisions (i.e. of pressure) on the decay of the revivals amplitudes, that can be deduced from the comparison of Figs. $\neq 2 \mathrm{a}$ and $12 b$, is significant. Furthermore, the comparison of the results in Figs. $12 b$ and $12 c$ gives a first indication of the quality of the ECS quantum model. Note that the permanent alignment is here negligible due to the weak intensity of the pulse (recall that, for moderate intensities, the magnitude of the permanent alignment scales with the square of the intensity while those of the revivals increase linearly 5 ). 


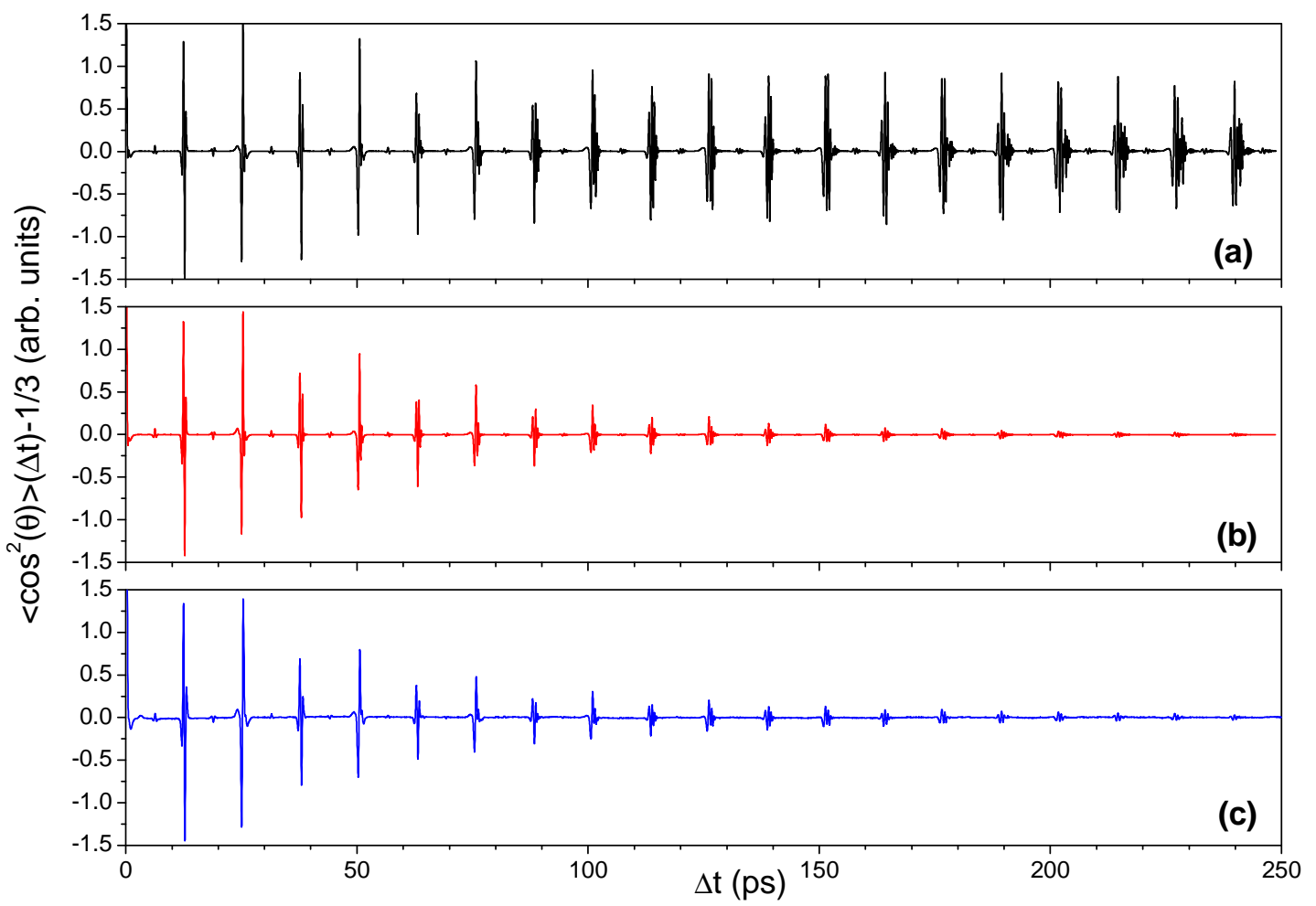

Fig. 2: Alignment factors predicted under collision-free conditions (a) and at a pressure of 0.69 bar (b) together with the measured values at 0.69 reproduced from Fig. 2 of Ref. 26 (c).

The quality of the ECS model for the dissipation of the alignment revivals is confirmed by Fig. 3 which displays detailed views of measured and computed alignment factors around some of the revivals. Both the shapes and the magnitudes are quite well reproduced by theory (note the large change of the y-axis scale from Fig. 3a to Fig. 3f) which slightly overestimates the decay with time of the revivals peak to peak amplitude. Let us note that one must be cautious when drawing conclusions on the model quality by a direct comparison, such as the one displayed in Fig. 3, between one single experimental recording of the alignment and the predictions for the following reasons: (i) the first is that measurements carry uncertainties, in particular because the intensity of the alignment pulse, as seen by the probe through the spatial overlapping of the two beams, may vary during the numerous shots needed to determine the alignment factor for different delays. Indeed, a slow drift in the pulse intensity induces a bias in the decay with time of the revivals amplitudes. (ii) The measured shapes and amplitudes of the alignment revivals are affected by the finite duration of the probe and non zero angle between the two pulses. These somehow act like a low-pass filter whose effects are taken into account in the calculation (see Sec. II.C) by convoluting the predicted timedependent alignment factor by a Gaussian function. This is obviously approximate and 
induces errors that affect the shape of the calculated alignment within the revivals (all the more that the revival shows numerous and narrow structures). According to these elements, carrying an analysis of the detailed differences between measured and calculated results in Fig. 3 and drawing conclusions from a comparison for a single alignment trace and pressure is, in our opinion, risky. However, the analysis of numerous experimental results for different pressures enables to deduces 12 a density normalized time constant for the decay of the amplitude of the alignment revivals. As shown by the values given in Table 1, the ECS model underestimates this time constant, a result which is consistent with what is indicated by Fig. 3, i.e. the fact that calculations overestimate the decay of the revivals amplitude with increasing delay.

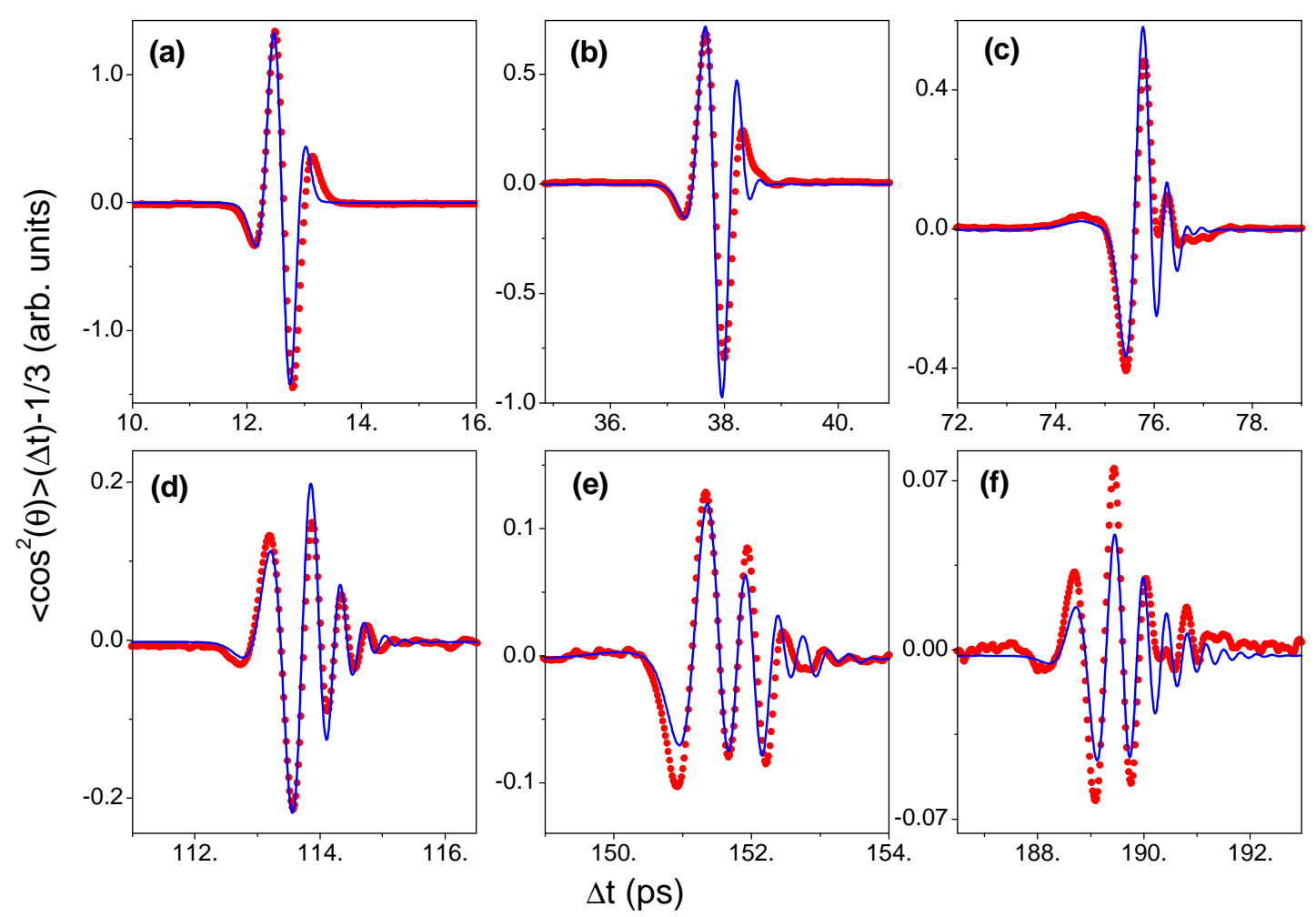

Fig. 3: Comparison between the measured (red circles, from the experiments of Ref. 26) alignment factor at 0.69 bar and $295 \mathrm{~K}$ and that predicted using the ECS model (blue line). Panels $(a),(b),(c),(d),(e)$, and $(f)$ display the results obtained around the revivals centered at $T_{\mathrm{rev}} / 2$, and $3 T_{\mathrm{rev}} / 2, \quad 6 T_{\mathrm{rev}} / 2, \quad 9 T_{\mathrm{rev}} / 2, \quad 12 T_{\mathrm{rev}} / 2$ and $15 T_{\mathrm{rev}} / 2$, respectively $\left(T_{\text {rev }}=(2 B c)^{-1}=25.1 \mathrm{ps}\right.$ being the rotational period $)$.

\section{B. Dissipation of the permanent alignment}


A comparison between measured alignment factors recorded at pressures of 2 and 6 bar for more intense laser pulses which make the permanent alignment significant and measurable and the associated predictions is plotted in Fig. 4. As can be seen, the quantum ECS model quite well reproduces the decays of the permanent alignment with time, confirming the interest of this approach for the modeling of the changes of the rotational angular momentum magnitude as well as orientation. Note that the apparent inconsistency in the differences between measurements and computations at 2 and 6 bar results from experimental errors (likely mostly on the zero level of the signal) and not from the fact that the model does not well describe the effect of increasing pressure. Indeed, while the time constant of the predicted exponential decay of the permanent alignment is inversely proportional to pressure, as it should be for the considered moderate pressures for which collisions are binary, the experimental values deviate from this rule. However, these inconsistencies are within the uncertainties of the measurements and scatter of the results obtained for various pressures which can be seen in Fig. 5 of Ref. 12 and lead to the error bars given in the Table 1. 


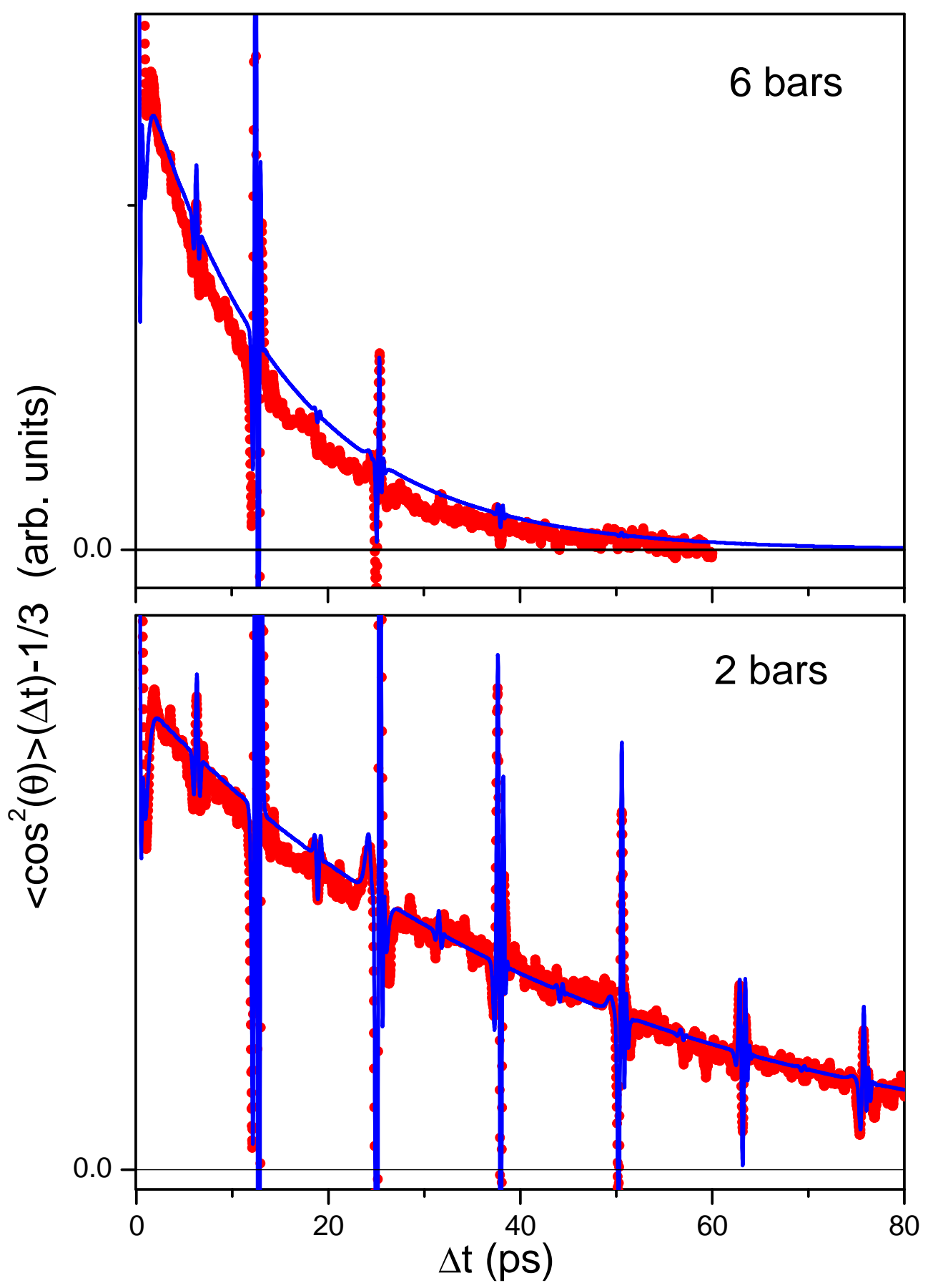

Fig. 4: Measured (red circles, from the experiments of Ref. 12) and computed (blue lines) alignment factors in pure ethane initially and $295 \mathrm{~K}$ and pressures of 2 bar (lower panel) and 6 bar (upper panel).

\section{C. "Gyroscopic" effect}

In order to go further, we determined from the ECS-computed alignment traces, the density normalized time constants driving the collisional decays of the amplitudes of the permanent $\left(\tau_{\mathrm{P}}\right)$ and transient $\left(\tau_{\mathrm{T}}\right)$ components of the alignment, as done in Refs. 5,12. The 
values obtained are reported in Table 1 with those retrieved from experiments 12 and predictions of requantized Classical Molecular Dynamics Simulations ${ }^{14}$. As can be seen the overall consistency between experimental and computed values is quite satisfactory, particularly when considering that no parameter was adjusted in the calculations in order to improve the agreement. In fact, tests show that it is possible to simultaneously lower $\tau_{\mathrm{P}}$ and increase $\tau_{\mathrm{T}}$ by lowering the ECS parameter $\alpha$ while increasing $Q_{0}$ [see Eq. (13)] at the price of a slight degradation of the ECS-predicted broadening parameters displayed in Fig. 1, however well within experimental uncertainties.

The values in Table 1 also show that measurements as well as computations reveal that the collisional dissipation of the permanent component of the alignment is significantly slower than that of the revivals amplitude. A similar situation was observed and predicted for linear molecules5-7,11 and explained by a "gyroscopic effect"5 resulting from the fact that collisions, while they change the rotational speed (i.e. the value of $J$ ), tend to conserve the direction of the rotational angular momentum (i.e. the value of $M / J$ ). In the case of ethane, the situation is similar, as shown by the state-to-state rates plotted in Fig. 5, since the collisional cross sections for the $(J, K, M) \rightarrow\left(J^{\prime}, K, M^{\prime}\right)$ change have a maximum for $\mathrm{M}^{\prime} / \mathrm{J}^{\prime} \approx \mathrm{M} / \mathrm{J}$. Note that, as discussed in Sec. II.C, the $\mathrm{C}_{2} \mathrm{H}_{6}-\mathrm{C}_{2} \mathrm{H}_{6}$ intermolecular potential is close to that for a pair of linear molecules. It is thus not surprising that Fig. 5 looks very similar to Fig. 9 of Ref. 5 which was obtained for pure $\mathrm{CO}_{2}$ gas.

\begin{tabular}{|l|c|c|}
\hline & $\begin{array}{c}\tau_{\mathrm{P}} \\
\text { (ps. amagat) }\end{array}$ & $\begin{array}{c}\tau_{\mathrm{T}} \\
\text { (ps. amagat) }\end{array}$ \\
\hline Exp12 & $67.6 \pm 6.4$ & $47.4 \pm 3.6$ \\
\hline Calc ECS & 83.0 & 40.7 \\
\hline Calc rCMDS14 & 72.8 & 44.9 \\
\hline
\end{tabular}

Table 1: Density-normalized time constants of the collision-induced decay of the amplitudes of the permanent $\left(\tau_{\mathrm{P}}\right)$ and transient $\left(\tau_{\mathrm{T}}\right)$ alignments. 

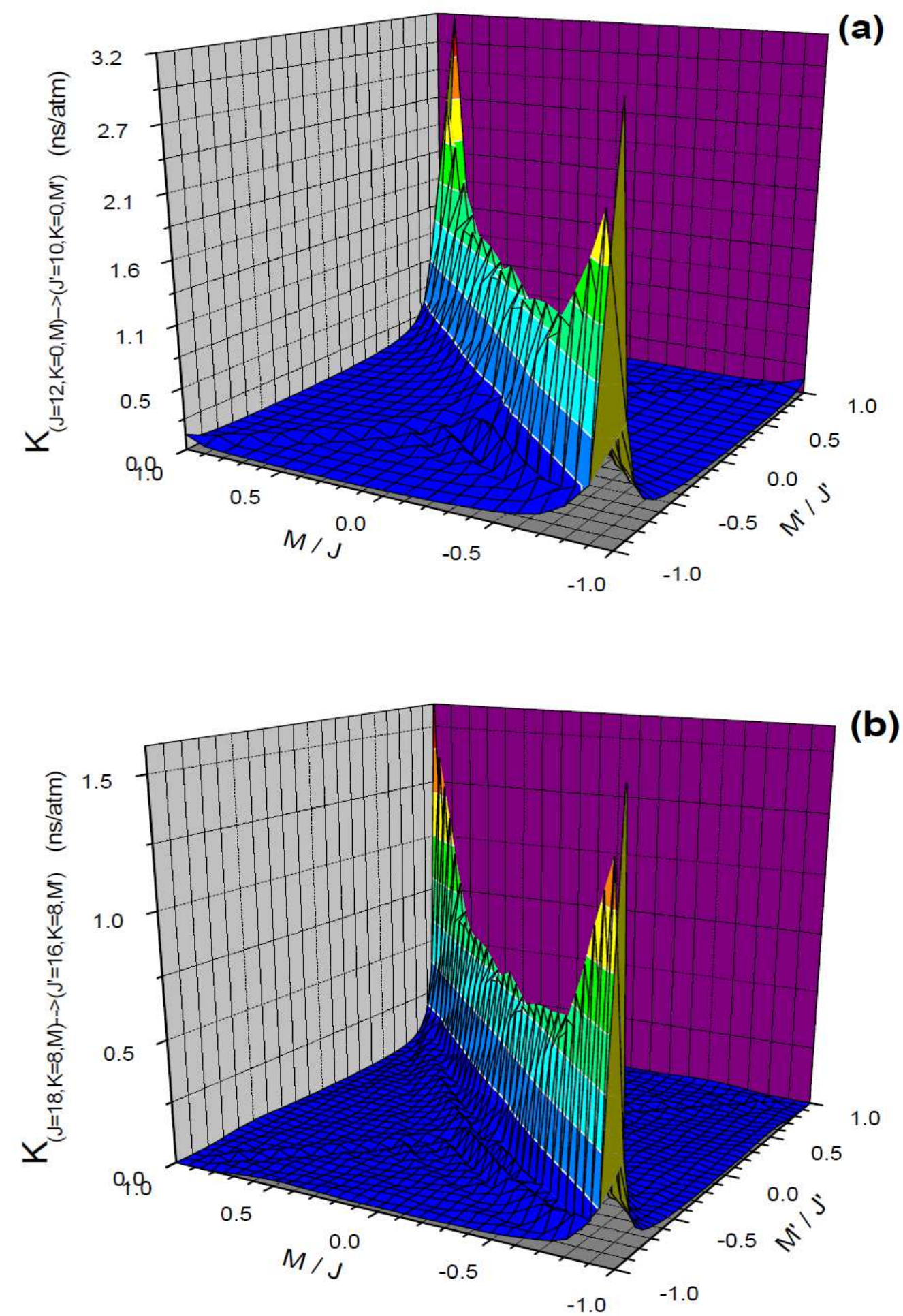

Fig. 5: Pressure normalized rates $K_{J, K, M \rightarrow J^{\prime}, K, M^{\prime}}$ versus $M / J$ and $M^{\prime} / J^{\prime}$ for $J=12, K=0$, $J^{\prime}=10(a)$ and for $J=18, K=8, J^{\prime}=16(b)$.

\section{Discussion on the models}

For a system like pure ethane at room temperature, rigorous "first principle" quantum mechanical methods cannot be used to predict the decay of the alignment even though an 
accurate intermolecular potential is available. ${ }^{25}$ The reason for this is that the number of significantly populated rotational states and of collisionally opened channels that need to be taken into account, as well as that of the state-to-state rates that are needed are by far too large for calculations to be tractable yet. One thus has to rely on approximate models. From this point of view, only the ECS approach described in this paper and the requantized Molecular Dynamics Simulations (rCMDS) presented in Ref. 14 have been proposed so far, which have different interests and weaknesses. The rCMDS have the advantages of directly using an input intermolecular potential, of taking into account the rotation-translation transfers and thus the eventual influence of the progressive heating of the translational motion during the collisional dissipation, and of correctly treating (at least classically) molecular pairs. In contrast, the ECS approach relies on knowledge of the basic rates which in many cases cannot be calculated and are approximately modeled (e.g. Eq. (13)] with parameters for the determination of which some measurements (e.g. Fig. 1) are needed. Furthermore, rotation-translation transfers are disregarded and the collision partner is treated as an atom with not rotational degree of freedom. However, within these approximations, the ECS approach is quantum and thus correctly describes the Raman transitions induced by the pump laser and the coherences in the wave-packet that they generate. Furthermore, going beyond the rigid rotor model and taking into account effects such as centrifugal distortion with this model is quite straight forward. In contrast, the requantization of classical simulations as proposed in Refs. 5 and 14 remains some kind of "trick" that is not fully satisfactory and has its limits, particularly when the kinetic temperature becomes comparable with the rotational constants. Furthermore, it is not possible to correctly introduce centrifugal distortion effects. Even though the collisional decay of the alignment may be well predicted within a rigid rotor approach, the shapes of the revivals are not correctly predicted as shown in Ref. 14. Hence the ECS and rCMDS have different interests and drawbacks but they are complementary. In order to explain this complementarity, let us consider the case where one needs to predict the dissipation of the alignment of a pure gas of linear or symmetric top molecules, for instance. If only an intermolecular potential is available, with not other data from which the ECS basic rates can be determined, rCMDS is the only solution. On the opposite, if there is no potential available but some line broadening measurements have been made that can be used for the determination of the ECS parameters, rCMDS cannot be made and the ECS model is the only applicable one. 


\section{CONCLUSION}

This paper presents the first quantum model for calculations of the collisional dissipation of the laser-induced alignment of a gas of symmetric-top molecules. It is based on a kinetic equation describing the time evolution of the density matrix, in which the effects of intermolecular collisions are taken into account through state-to-state rates constructed using the Energy Corrected Sudden approximation. This approach is applied to ethane gas for which measurements of the alignment have been made recently. The associated comparison between predicted and measured time-dependent alignment signals demonstrates the quality of the model for the decays of both the permanent and transient components of the alignment. The analysis of the computed state-to-state collisional rates shows that they are the largest when the orientation of angular momentum associated with the rotation of the molecule symmetry axis is conserved (i.e. $M / J$ practically does not change). This explains the experimentally and theoretically observed fact that the permanent alignment decays more slowly than do the alignment revivals amplitudes. It is important to note that the present paper only provides a limited test of the ECS model for the collisional dissipation of symmetric-tops molecules. Indeed, as discussed in this paper, $\Delta K \neq 0$ transitions play a negligible role in the case of pure ethane whose behavior is thus close to that of a linear molecule. It would thus be of interest to find a molecular system (including a symmetric-top molecule and an eventually different one in which it is diluted) for which the situation is different and that could be investigated experimentally. This may not be so easy since two constraints should be satisfied. The first is that the sensitivity of intermolecular forces to rotation of the molecule around its symmetry axis should be important. The second is that the anisotropy of the polarizability of the molecule should be sufficient to enable a significant degree of alignment using current and reasonable laser pulse intensities. In any case, one should remember that the proposed ECS model has limitations that may not make it suitable for more complex systems than pure ethane, for two main reasons. The first is that the collision partner is treated as an atom so that resonance effects (appearing when each molecule of the colliding pair makes a rotational change) occurring in molecule-molecule collisions are not taken into account, The second is the use of approximate corrections of the IOS model, through the adiabaticity factor, in order to take into account the fact that molecules do rotate during collisions. These have moderate consequences when the rotational constant is small but may lead to significant errors when it is not the case (e.g. $\mathrm{NH}_{3}, \mathrm{PH}_{3}, \mathrm{CH}_{3} \mathrm{D}$ or linear molecules such as $\mathrm{HCl}$ and $\mathrm{HF}$ ). 
Acknowledgements: This work was supported by the Conseil Régional de Bourgogne under the PhotCom PARI program, the European Union (FEDER program), the Labex ACTION program (No. ANR-11-LABX-01-01). 


\section{References}

1. H. Stapelfeldt and T. Seideman, Rev. Mod. Phys. 75, 543 (2003).

2. T. Seideman and E. Hamilton, Adv. At., Mol., Opt. Phys. 52, 289 (2005).

3. S. Ramakrishna and T. Seideman, Phys. Rev. Lett. 95, 113001 (2005).

4. S. Ramakrishna and T. Seideman, J. Chem. Phys. 124, 034101 (2006).

5. J.-M. Hartmann and C. Boulet, J. Chem. Phys. 136, 184302 (2012).

6. T. Vieillard, F. Chaussard, F. Billard, D. Sugny, O. Faucher, S. Ivanov, J.-M. Hartmann,

C. Boulet, and B. Lavorel, Phys. Rev. A 87, 023409 (2013).

7. F. Chaussard, T. Vieillard, F. Billard, O. Faucher, J.-M. Hartmann, C. Boulet, and B. Lavorel, J. Raman Spectrosc. 46, 691 (2015).

8. J.-M. Hartmann, C. Boulet, T. Vieillard, F. Chaussard, F. Billard, O. Faucher, and B. Lavorel, J. Chem. Phys. 139, 024306 (2013).

9. T. Vieillard, F. Chaussard, D. Sugny, B. Lavorel, and O. Faucher, J. Raman Spectrosc. 39, 694 (2008).

10. N. Owschimikow, F. Konigsmann, J. Maurer, P. Giese, A. Ott, B. Schmidt, and N. Schwentner, J. Chem. Phys. 133, 044311 (2010).

11. S. Fleischer, Y. Zhou, R. W. Field, and K. A. Nelson, Phys. Rev. Lett. 107, 163603 (2011).

12. H. Zhang, F. Billard, X. Yu, O. Faucher, and B. Lavorel, J. Chem. Phys. 148, 124303 (2018).

13. R. Damari, D. Rosenberg, and S/ Fleischer, Phys. Rev. Lett. 119, 033002 (2017).

14. J.-M. Hartmann, C. Boulet, H. Zhang, F. Billard, X. Yu, O. Faucher, and B. Lavorel, J. Chem. Phys 149, 154301 (2018).

15. V. May and O. Kuhn. Charge and Energy Transfer Dynamics in Molecular Systems, Wiley, Weinheim (2004).

16. P. Bréchignac, A. Picard-Bersellini, R. Charneau, and J.-M. Launay, Chem. Phys. 53, 165 (1980).

17. A.E. DePristo, S.T. Augustin, R. Ramaswamy, and H. Rabitz, J. Chem. Phys. 71, 850 (1979).

18. J.-M. Hartmann, C. Boulet, D. Robert. Collisional Effects on Molecular Spectra: Laboratory experiments and models, consequences for applications. Elsevier, Amsterdam (2008).

19. S. Green, J. Chem. Phys. 70, 816 (1979).

20 R.B. Bernstein editor. Atom-molecule theory: A Guide for the Experimentalist. Plenum Press, New York, (1979).

21 R. Goldflarn, S. Green, and D. J. Kouri, J. Chern. Phys. 67, 4149 (1977).

22. J. Koubek, C. Boulet, A. Perrin, Š. Urban and J.-M. Hartmann, J. Mol. Spectrosc. 266, 12 (2011).

23. S. Hadded, F. Thibault, P.-M. Flaud, H. Aroui, and J.-M. Hartmann, J. Chem. Phys. 116, 7544 (2002;).

24. S. Hadded, F. Thibault, P.-M. Flaud, H. Aroui, and J.-M. Hartmann, J. Chem. Phys. 120, 217 (2004).

25. R. Hellmann, J. Chem. Eng. Data 63, 470 (2018).

26. H. Zhang, F. Billard, O. Faucher, and B. Lavorel, J. Raman Spectrosc. 49, 1350 (2018) 
27. T.A. Brunner and D. Pritchard. Fitting laws for rotationally inelastic collisions. In Dynamics of the Excited State, K.P. Lawley Ed., p. 589, Wiley, NY (1982).

28. V. M. Devi, D. C. Benner, C.P. Rinsland, M.A.H. Smith, R.L. Sams, T.A. Blake, J.-M. Flaud, K. Sung, L.R. Brown, and A.W. Mantz, J.. Quant. Spectrosc. Radiat. Transf. 111, 2481 (2010). 